\title{
TRANSTORNO DE PERSONALIDADE BORDERLINE, SINTOMAS DISSOCIATIVOS E MEMÓRIA
}

\section{ARTIGO DE REVISÃO}

SILVA, Fernanda Vicilli Souza ${ }^{1}$, PALMA, Priscila de Camargo ${ }^{2}$

SILVA, Fernanda Vicilli Souza. PALMA, Priscila de Camargo. Transtorno de personalidade Borderline, sintomas dissociativos e memória. Revista Científica Multidisciplinar Núcleo do Conhecimento. Ano 06, Ed. 07, Vol. 01, pp. 140-154. Julho de 2021. ISSN: 2448-0959, Link de acesso: https://www.nucleodoconhecimento.com.br/psicologia/sintomas-dissociativos, DOI: 10.32749/nucleodoconhecimento.com.br/psicologia/sintomas-dissociativos

\section{RESUMO}

Este estudo aponta a relação entre o Transtorno de Personalidade Borderline (TPB), sintomas dissociativos e memória. Os sintomas dissociativos - que são caracterizados pele perturbação e/ou descontinuidade da integração normal de consciência, memória, identidade, emoção, percepção, representação corporal, controle motor e comportamento -, dos quais fazem parte as alterações de memória, foram incluídos nos critérios diagnósticos do TPB apenas no Manual Diagnóstico e Estatístico de Transtornos Mentais (DSM) IV, tendo sido mantido no DSM 5, e ainda existem poucos estudos sobre a prevalência destes sintomas no transtorno e sua influência. A dissociação relacionada ao estresse ocorre em 75 a 80\% dos pacientes com TPB. Foi realizada revisão bibliográfica com leitura, análise e interpretação de material com

\footnotetext{
1 Especialização em Terapia Cognitivo Comportamental, MBA em Gestão de Pessoas e Desenvolvimento Humano, Formação em Terapia do Esquema (cursando), Graduação em Psicologia.
}

${ }^{2}$ Orientadora. Doutora em Psicologia. 
abordagens relacionadas ao tema de estudo. Este artigo apresenta diferentes definições da dissociação, desde a interpretação psicanalítica até a visão neurobiológica e ainda as principais formas de tratamento na atualidade - as derivadas da Terapia Cognitivo Comportamental: Terapia Comportamental Dialética e Terapia do Esquema.

Palavras-chave: borderline, dissociação, memória, tratamento, personalidade.

\section{INTRODUÇÃO}

Norberto Bobbio (1997, p. 30) aponta que "Dizemos: afinal, somos aquilo que pensamos, amamos, realizamos. E eu acrescentaria: somos aquilo que lembramos". A construção da identidade do indivíduo é um processo contínuo e multifatorial que engloba, entre outros, a vivência do sujeito, sua genética e suas escolhas. O presente artigo abordará a visão da Terapia Cognitivo Comportamental sobre o Transtorno de Personalidade Borderline, bem como as diretrizes para tratamento de acordo com esta abordagem. Além disso, será abordada uma perspectiva relacionada aos aspectos que tornam o indivíduo único devido às alterações que apresentam: transtorno de personalidade e memória, relacionadas a sintomas dissociativos.

Tal estudo visa ainda entender o papel da dissociação no Transtorno de Personalidade Borderline, além de identificar as alterações de memória relacionadas a dissociação e elencar as principais formas de tratamento na atualidade. Para isso, será feita uma revisão abrangente das diferentes definições da dissociação, desde a interpretação psicanalítica até a visão neurobiológica, assim como abordadas as principais formas de tratamento na atualidade - as derivadas da Terapia Cognitivo Comportamental: Terapia Comportamental Dialética e Terapia do Esquema. (CORDIOLI, 2019)

A dissociação é caracterizada por "perturbação e/ou descontinuidade da integração normal de consciência, memória, identidade, emoção, percepção, representação corporal, controle motor e comportamento" (APA, 2014). Pode-se dizer que sua 
presença altera além da percepção sobre si mesmo, o mundo e os outros, a individualidade do ser.

O acervo de nossas memórias faz com que cada um de nós seja o que é: um indivíduo, um ser para o qual não existe outro idêntico. Alguém poderia acrescentar: '...e também somos o que resolvemos esquecer'. Sem dúvida; mas não há como negar que isso já constitui um processo ativo, uma prática da memória: nosso cérebro "lembra" quais são as memórias que não quer trazer à tona, e evita recordá-las: as humilhações, por exemplo, ou as situações profundamente desagradáveis ou inconvenientes. De fato, não as esquece, pelo contrário: as lembra muito bem e muito seletivamente, mas as torna de difícil acesso. (IZQUIERDO, 2014, p. 14)

A personalidade, da mesma forma, torna o indivíduo único. Cada transtorno psiquiátrico tem seus critérios diagnósticos e características fundamentais, mas nunca é o mesmo para dois indivíduos.

Segundo a APA (2014), o Transtorno de Personalidade Borderline é caracterizado por um padrão difuso de instabilidade das relações interpessoais, da autoimagem e dos afetos e de impulsividade acentuada que surge no início da vida adulta e está presente em vários contextos. Pode-se também encontrar no DSM V que há, entre outros, esforços desesperados para evitar abandono real ou imaginado; recorrência de comportamento, gestos ou ameaças suicidas ou de comportamento automutilante; acentuada reatividade de humor; sentimentos crônicos de vazio e raiva intensa e inapropriada ou dificuldade em controlá-la.

De acordo com Scalabrini (2016), um dos critérios diagnósticos do TPB é a presença de sintomas dissociativos intensos, que são presentes nos transtornos psiquiátricos em um continuum, sendo que a incidência no TPB é menor apenas do que nos Transtornos Dissociativo de Identidade e de Estresse Pós Traumático.

Os sintomas dissociativos alteram não apenas a individualidade do sujeito, mas também a forma de seu tratamento psicológico, isto porque, independente de qual é o transtorno envolvido (TPB, TDI, TEPT, Depressão, etc.), a integração das informações é afetada; dissociada. É necessária a estruturação de um tratamento personalizado para potencializar os efeitos da psicoterapia e torná-los mais efetivos. 
Com o objetivo de abordar a relação entre o Transtorno de Personalidade Borderline, sintomas dissociativos e memória, este estudo analisou a bibliografia disponível nas plataformas PubMed, SCIELO e Google Acadêmico.

\section{DESENVOLVIMENTO}

A psicologia da personalidade, segundo Friedman e Schustack (2004), é o estudo científico das forças psicológicas que tornam as pessoas únicas. $O$ autor diz ainda que a personalidade tem oito aspectos principais que, reunidos, ajudam-nos a compreender a natureza complexa do indivíduo; são eles: aspectos inconscientes; forças do ego; biologia; condicionamento e modelagem; cognição; traços, habilidades e predisposições específicos; aspecto espiritual e interação entre o indivíduo e determinado ambiente.

Para fins diagnósticos, um transtorno da personalidade é definido como

um padrão persistente de experiência interna e comportamento que se desvia acentuadamente das expectativas da cultura do indivíduo, é difuso e inflexível, começa na adolescência ou no início da fase adulta, é estável ao longo do tempo e leva a sofrimento ou prejuízo. (APA, 2014, p. 645)

O Transtorno de Personalidade Borderline (TPB) é caracterizado por notável instabilidade nas relações interpessoais, na autoimagem e nos afetos, com impulsividade acentuada e é relativamente comum - 1,6 a 5,9\% da população (APA, 2014), sendo cerca de 70\% mulheres (BECK; DAVIS e FREEMAN, 2017). De acordo com Krause (2017a), a dissociação relacionada ao estresse ocorre em 75 a 80\% dos pacientes com TPB.

É interessante notar que além de os sintomas dissociativos integrarem os critérios diagnósticos para o TPB, o Transtorno Dissociativo de Identidade e o Transtorno de Personalidade Borderline são comórbidos: 10 a $27 \%$ da população borderline pode ser diagnosticada com TDI e 30 a 70\% da população TDI pode ser diagnosticada com TPB (KORZEKWA et al, 2009). 
Dentre os critérios diagnósticos do TPB no DSM 5 (APA, 2014) estão os sintomas dissociativos intensos, tendo sido este critério incluído apenas no DSM IV. Possivelmente por ser o critério mais recente, hajam poucos estudos relacionando sintomas dissociativos e Transtorno de Personalidade Borderline. Tais temas são encontrados juntos, de maneira geral, quando se fala em trauma, abuso sexual, TDI ou TEPT.

A dissociação é caracterizada por perturbação e/ou descontinuidade da integração normal de consciência, memória, identidade, emoção, percepção, representação corporal, controle motor e comportamento. Os sintomas dissociativos podem potencialmente perturbar todas as áreas do funcionamento psicológico. (APA, 2014, p. 291)

De acordo com o DSM 5, sintomas dissociativos são vivenciados como intrusões espontâneas na consciência e no comportamento, acompanhadas por perdas de continuidade na experiência subjetiva ou incapacidade de acessar informações e de controlar funções mentais que normalmente são de fácil acesso ou controle.

Korzekwa et al. (2009) explicam que além da desrealização, da despersonalização e da amnésia, os sintomas dissociativos relativos ao TPB podem ser relacionados à dissociação somatoforme, que inclui anestesia, paralisia, dor inexplicável e movimentos incontroláveis. Além disso, nota-se que indivíduos com Transtorno de Personalidade Borderline e sintomas dissociativos apresentam alterações relativas à temporalidade e identidade (FUCHS, 2007) e confusão entre sonho e realidade (SKRYPINSKA e SZMIGIELSKA, 2015).

Segundo Skrypinska e Szmigielska (2015), a cisão do self está relacionada ao TPB, incluindo a fragmentação temporal, e evita a necessidade de tolerar a ambiguidade e a incerteza nas relações interpessoais, mas ao preço de um sentimento de vazio crônico causado pela inabilidade de integrar passado, presente e futuro e então estabelecer um senso coerente de identidade.

Enquanto isso, as variáveis que podem indicar uma tendência a experienciar a Confusão Sonho-Realidade (CSR) são: problemas com sono, dissociação, conteúdo negativo dos sonhos, distúrbios cognitivos, como por exemplo, alterações na 
metacognição e distúrbios de memória, além de limites estreitos entre o self e os outros e entre diferentes estados de consciência.

A presença de sintomas somatoformes remete aos estudos de Freud sobre a histeria e à presença de sintomas dissociativos como mecanismos de defesa. Para a teoria psicanalítica esse mecanismo é considerado proposital, ainda que inconsciente, podendo ser desencadeado por eventos específicos ou se apresentar como traço de personalidade. Já segundo Pierre Janet (1901), o fenômeno não teria origem proposital ou funcional, mas surgiria quando o indivíduo experiencia emoções veementes que levariam ao estreitamento do campo atencional e desorganização das funções usuais de integração das informações na consciência.

De acordo com Cardeña (1994), a dissociação como mecanismo de defesa poderia ter uma origem filogenética devido à adaptação conferida pela experiência dissociativa em situações traumáticas e a seu paralelo a comportamentos de passividade.

No modelo da teoria cognitiva da personalidade e psicopatologia desenvolvido por Beck (1996), a proposição central é que a dissociação é um produto da desvinculação de processos mentais, que pode ocorrer em vários estágios de processamento da informação. Tal desvinculação está associada, de maneira geral, a experiências traumáticas.

Trauma é um prejuízo, um estado psíquico ou comportamental desorientado, provocado por estresse mental ou emocional ou dano físico, relacionado a eventos que podem provocar medo agudo ou crônico. A exposição a eventos traumáticos ativa os sistemas neurais de resposta ao estresse (sistema nervoso central, sistema nervoso periférico, sistema neuroendócrino e sistema imunológico), particularmente 0 eixo hipotálamo-hipófise-adrenal $(\mathrm{HHA})$. (GARBARINO; KOSTELNY e DUBROW apud BORGES e DELL'AGLIO, 2008, p. 372)

A relação entre trauma e alterações cognitivas e neuropsicológicas tem sido estudada, principalmente em relação ao Transtorno de Estresse Pós-traumático (TEPT), sendo possível identificar mudanças estruturais e funcionais no cérebro. Estudos de neuroimagem (MRI e PET) tem apontado alterações no volume do hipocampo, da amígdala, do giro cingulado anterior e do córtex pré-frontal. Tais alterações podem 
resultar em prejuízos na memória de curto prazo, na memória declarativa, na atenção sustentada, na aprendizagem verbal, na construção visual e em funções executivas. (BORGES e DELL'AGLIO, 2008; PEC, 2018)

Já em 1998 Sierra e Berrios, apud Krause (2017b), propuseram que sintomas de despersonalização podem estar associados a desconexão córtico-límbica, envolvendo a amígdala, o córtex cingulado anterior e estruturas pré-frontais. É como se $o$ sistema afetivo desligasse.

Sabe-se que a amígdala está envolvida na detecção e processamento das emoções relacionadas ao estresse e a respostas de medo - estados de distanciamento podem estar associados a uma redução da reatividade desta área. Da mesma forma, diminuição na atividade da área occipito-temporal do córtex e da ínsula pode refletir a redução da consciência emocional/interoceptiva. (KENNEDY, 2004)

Ainda não é possível afirmar se as alterações supracitadas são causas ou consequências da psicopatologia, seja ela TPB ou TEPT. Apesar disso, a presença de experiência traumática durante a vida do sujeito é confirmada.

A dissociação teve seu papel na proteção do indivíduo em situações de estresse grave ou crônico, porém pode se tornar mal adaptativa e prejudicar o tratamento. Pacientes muito vulneráveis a experiências peritraumáticas (nas quais a dissociação ocorre durante o evento traumático) tendem a responder de maneira similar a outros eventos ao longo da vida - indivíduos que aprenderam a dissociar em resposta a situações traumáticas/estressantes têm uma maior tendência a fazer o mesmo na presença de estressores menores. (KRAUZE, 2017a; NEGRO JUNIOR, 1999)

Em relação ao tratamento, o ideal é a utilização de psicoterapia associada ao uso de fármacos.

Atualmente a primeira opção de recurso terapêutico para o Transtorno de Personalidade Borderline é a Terapia Comportamental Dialética. Segundo Marsha Linehan (2017), a DBT é uma terapia cognitivo-comportamental que foi desenvolvida 
originalmente para indivíduos cronicamente suicidas, diagnosticados com transtorno da personalidade borderline (TPB).

A importância de poder contemplar a necessidade de mudança e, ao mesmo tempo, a de validação da experiência psíquica individual deu origem à DBT .

"Como o nome diz, a dialética é uma parte essencial da terapia, sendo preciso, por parte do terapeuta, aceitar o paciente como ele é e buscar mudanças significativas em sua maneira de agir e viver." (CORDIOLI, 2019, p. 215)

Para Linehan (2017), as metas de tratamento da DBT, em ordem de prioridade, são: abordar comportamentos que ameaçam a vida, comportamentos que interferem na terapia, comportamentos que prejudicam ou interferem na qualidade de vida e aquisição de habilidades.

Outra opção de tratamento é a Terapia de Esquema. De acordo com Sajadi (2015), a TE surgiu a partir da dificuldade de adesão à terapia de pacientes com transtornos crônicos e de personalidade, integrando vários modelos de psicoterapia, como as abordagens cognitivo-comportamental, construtivista, psicodinâmica, de relações objetais e da Gestalt.

A Teoria do Apego tem papel importante na TE, sendo base para o entendimento da formação e manutenção dos Esquemas Iniciais Disfuncionais (EIDs). Os Modos Esquemáticos são padrões característicos de funcionamento do indivíduo, que foram adaptativos em dado momento, mas que não são mais. O modo esquemático criança zangada é relacionado às EIDs abandono, desconfiança/abuso, privação emocional e subjugação, esquemas relacionados ao transtorno de personalidade borderline.

De acordo com a teoria de Bowlby, o apego inseguro/ansioso tem uma tendência ao temperamento com afeto negativo e maior propensão à personalidade borderline, paranoide, histriônica, esquiva e traços dependentes.

O objetivo da Terapia do Esquema é que as necessidades emocionais básicas do paciente possam ser supridas, na maior extensão possível, por meio do 
relacionamento terapêutico e de técnicas e exercícios experienciais, cognitivos e comportamentais, dentro e fora do setting terapêutico.

A hipótese norteadora deste estudo é que pacientes com TPB apresentam alto grau de dissociação e consequentes alterações de memória, que têm influência no curso e tratamento do transtorno.

\section{MÉTODO}

Este estudo conta com uma revisão bibliográfica que constitui a base teórica para o desenvolvimento de todo trabalho de investigação em ciência, abrangendo toda a literatura tornada pública, e selecionada em relação ao tema estudado.

A coleta das informações para esta pesquisa decorre da investigação em 12 livros técnicos científicos e 12 artigos científicos publicados em bases de dados eletrônicas como PUBMED, SCIELO e GOOGLE ACADÊMICO.

A seleção dos artigos acontece a partir da utilização das palavras-chave borderline, dissociação e memória. Os critérios de inclusão abrangem artigos nacionais e internacionais, em Português e Inglês, publicados entre 2015 e 2020.

\section{RESULTADOS E DISCUSSÃO}

Segundo Friedman e Schustack (2004), todas as abordagens cognitivas da personalidade têm em comum a visão de que a essência da personalidade se encontra na maneira como as pessoas pensam, isto é, no modo como compreendemos os acontecimentos no nosso mundo, na maneira como apreendemos a natureza e as ações de outras pessoas, em como aprendemos em nossos ambientes sociais e no modo como controlamos e compreendemos nossos próprios comportamentos.

De acordo com Wainer (2016), as metas de tratamento do TPB podem ser definidas como: reduzir os sintomas do transtorno (conflitos nos relacionamentos, medo do abandono, problemas de identidade, instabilidade emocional e vazio); sentir-se seguro 
com a experiência e expressão de emoções, necessidades e o vínculo pessoal com os outros; ajudar o paciente a crescer e se desenvolver tornando-se independente e maduro; desenvolver uma vida gratificante nos níveis pessoal e social; estabelecimento de limites para comportamentos impulsivos e inadequados; identificar as crenças centrais e seu efeito no afeto, motivação e comportamento.

Segundo Cordioli (2019), existe ainda uma abordagem hierárquica para decidir a agenda, sendo: 1. Comportamentos que ameaçam a vida; 2 . Comportamentos que interferem na terapia; 3. Comportamentos que prejudicam ou interferem na qualidade de vida; e 4. Aquisição de habilidades. O foco no relacionamento terapêutico é característico do tratamento de transtornos de personalidade pela TCC.

Para Beck, Davis e Freeman (2017), na formulação Beckiana do Transtorno de Personalidade Borderline, há três pressupostos fundamentais no transtorno: "O mundo é perigoso e maligno", "Sou impotente e vulnerável", e "Sou inerentemente inaceitável". Além disso, existem outras duas características cognitivas cruciais: o pensamento dicotômico, oscilando entre pressupostos de dependência e pressupostos paranóides que alternam entre apegar-se excessivamente aos outros e afastar-se das pessoas por desconfiança, e uma noção fraca de identidade.

É sugerido o uso de técnicas experienciais no tratamento, notadamente o trabalho com imagens mentais. A importância dos métodos experienciais das primeiras memórias da infância é enfatizada, associados ao "aqui e agora". (BECK; DAVIS e FREEMAN, 2017)

Para o tratamento pelo modelo de modos de esquema de Young, segundo Wainer (2016), as crenças centrais típicas, no modo criança abandonada, são: "Você é ruim (mal) e merece castigo", "Seus desejos/opiniões/emoções são infundados", "Você não tem o direito de expressar seus desejos/opiniões/emoções" e "Você está manipulando". Assim sendo, o indivíduo com frequência não somente experiencia estes pensamentos punitivos, mas agrega a eles atos punitivos, como automutilar-se. A culpa é o sentimento proeminente. 
Há ainda os comportamentos relacionados ao modo criança zangada, onde as crenças subjacentes são: "Meus direitos básicos foram-me negados", "As outras pessoas são mesquinhas e más" e "Preciso lutar ou simplesmente pegar o que preciso para sobreviver". (BECK; DAVIS e FREEMAN, 2017)

\begin{abstract}
A terapia baseada no modelo de Young visa reduzir o uso do modo protetor desligado, abandonar o modo criança zangada/abusada pelo oferecimento de formas saudáveis de assertividade, expulsar o modo pai punitivo do sistema do paciente e aumentar a força do modo adulto saudável. (BECK; DAVIS e FREEMAN, 2017, p. 309)
\end{abstract}

Para tanto, são utilizadas técnicas como a auto revelação, a reparentalização (para promover um apego seguro) e a confrontação empática.

A Terapia Comportamental Dialética (DBT) considera que direcionar o tratamento de pacientes borderline apenas para a mudança pode trazer muito desconforto, causando a constante sensação de invalidação. Desta forma, considera a importância da validação da experiência psíquica individual. (CORDIOLI, 2019)

Ainda de acordo com Cordioli (2019), as estratégias nucleares da DBT são a validação e a resolução de problemas, além de equilibrar a responsabilização do paciente por desenvolver habilidades ou alcançar melhoras, sendo o terapeuta um consultor.

Segundo Cordioli (2019) algumas das técnicas utilizadas nesta abordagem são: uso de metáforas, advogado do diabo e ativar a mente sábia.

A DBT faz ainda uso de técnicas de mindfulness, de acordo com Cordioli (2019). Tais técnicas são usadas não somente para o tratamento do TPB, mas também para tratar a dissociação. Segundo Forner (2018) "a dissociação é preservar a vida e a atenção plena parece melhorar a nossa vida". A atenção plena envolve atividades interconectadas nas redes cerebrais que facilitam a autoconsciência e a regulação emocional.

Gonzalez (2014) esclarece a importância de, no tratamento da dissociação, abordar especificamente a fragmentação. Estudos empíricos mostram que não abordar 
diretamente a fragmentação da personalidade e os sintomas dissociativos contribui para uma piora do prognóstico.

\begin{abstract}
Se partirmos da teoria da dissociação estrutural, a fragmentação da personalidade pelo trauma é o mecanismo central dos transtornos dissociativos e de todos os quadros de base traumática. Portanto, atuar no sentido de reparar essa fragmentação deverá ser o elemento focal da terapia. Para isso, é preciso levar em conta que existem diferentes estados mentais no paciente, então, devemos utilizar técnicas específicas que nos permitam conduzir esses aspectos. (GONZALEZ, 2014, p. 50)
\end{abstract}

De acordo com Cordioli (2019), a dessensibilização e reprocessamento por movimentos oculares (EMDR) pode ser compreendida pela psicologia cognitiva, pois considera o modo como o indivíduo processa informações e como as memórias são armazenadas e recodificadas, tanto no cérebro quanto no corpo.

"Podemos fazer um trabalho cognitivo sobre as distorções graves apresentadas por esses pacientes, entendendo como são geradas e quais distintos estados de consciência podem apresentar diferentes distorções cognitivas." (GONZALEZ, 2014, p. 51)

Gonzalez (2014) coloca que as fases do tratamento com EMDR estão estabelecidas nas diretrizes clínicas da ISSD (Sociedade Internacional de Estudos da Dissociação). É necessária uma fase de estabilização e estabelecimento de segurança, uma segunda de trabalho com o trauma e, por último, um trabalho específico de integração das partes dissociadas. Não se pode desconsiderar a importância da relação terapêutica.

\title{
CONSIDERAÇÕES FINAIS
}

A alta prevalência de sintomas dissociativos e alterações de memória no Transtorno de Personalidade Borderline e sua influência sobre o curso e o tratamento do transtorno apontam para a necessidade de mais estudos sobre o assunto. A dissociação e a memória já vêm sendo estudadas desde o século XIX, através das histéricas e de Hermann Ebbinhaus, respectivamente, mas apenas em 1952 a 
dissociação - e consequentemente as alterações de memória - passou a ser considerada um critério diagnóstico para o TPB, sendo mantido no DSM 5.

Apesar disso, há poucos estudos sobre dissociação que não estejam relacionados ao Transtorno de Estresse Pós Traumático ou ao Transtorno Dissociativo de Identidade. Ainda, não foram encontrados artigos abordando o tratamento de tal sintoma.

A TCC e suas vertentes (DBT, Terapia do Esquema e Mindfulness) oferecem uma gama de protocolos de tratamento, bem como informações consistentes sobre o funcionamento cognitivo do indivíduo com TPB. O EMDR apresenta possibilidades de tratamento para a dissociação, trabalhando com reestruturação cognitiva.

Vale salientar que, independentemente da abordagem, a importância da relação terapêutica foi considerada em todos os materiais utilizados.

Sugere-se mais estudos sobre o tema, visto que o material sobre tal relação é escasso; além disso, que sejam feitas pesquisas sobre os meios de tratamento de pacientes com Transtorno de Personalidade Borderline e sintomas dissociativos, considerando-se a prevalência dos mesmos nesta psicopatologia.

\section{REFERÊNCIAS}

AMERICAN PSYCHIATRIC ASSOCIATION - APA. Manual diagnóstico e estatístico de transtornos mentais: DSM-5, 5ª ed. Porto Alegre: Artmed, 2014. $922 \mathrm{p}$.

BECK, Aaron Temkin. Beyond belief: a theory of modes, personality and psychopathology. In P. M. Salkovskis (Ed.), Frontiers of cognitive therapy (pp. 125). London: Guilford Press, 1996. 553 p.

BECK, Aaron; DAVIS, Denise; FREEMAN, Arthur. Terapia cognitiva dos transtornos da personalidade, $3^{3}$ ed. Porto Alegre: Artmed, 2017. 440 p.

BOBBIO, Norberto. O tempo da memória. Rio de Janeiro: Elsevier, 1997. 240 p. 
BORGES, Jeane Lessinger; DELL`AGLIO, Débora Dalbosco. Relações entre abuso sexual na infância, transtorno de estresse pós-traumático (TEPT) e prejuízos cognitivos. Psicol. estud. vol.13, n.2, pp.371-379. ISSN 1413-7372. 2008. Disponível em: https://doi.org/10.1590/S1413-73722008000200020. Acesso em 10/08/2020.

CARDEÑA, Etzel. The Domain of Dissociation. In: Lynn SJ, Rhue JW, editors. Dissociation: Clinical and Theoretical Perspectives (p.15-31). New York: The Guilford Press, 1994. $477 \mathrm{p}$.

CORDIOLI, Aristides Volpato; GREVET, Eugenio Horácio. Psicoterapias: Abordagens Atuais. Porto Alegre: Artmed, 2019. 792 p.

FORNER, Chrisine. What Mindfulness can learn about Dissociation and what Dissociation can learn from Mindfulness. Journal of Trauma \& Dissociation, JanFeb 2019;20(1) pp1-15. 10 de agosto de 2018. Disponível em: https://pubmed.ncbi.nlm.nih.gov/30095378/. Acesso em 11/08/2020.

FRIEDMAN, Howard; SCHUSTACK, Mirian. Teorias da personalidade: da teoria clássica à pesquisa moderna. Londres: Pearson, 2004. 574 p.

FUCHS, Thomas. Fragmented Selves: Temporality and Identity in Borderline Personality Disorder. Psychopathology, 40(6), pp.379-387. 25 de julho de 2007. Disponível em: https://pubmed.ncbi.nlm.nih.gov/17652950/. Acesso em 22/08/2020.

GONZALEZ, Anabel. Transtornos dissociativos: Diagnóstico e tratamento. Brasília: TraumaClinic Edições, 2014. 294 p.

IZQUIERDO, Ivan. Memória. Porto Alegre: Artmed, 2014. 124 p.

JANET, Pierre. The mental state of hystericals. New York: Putnan, 1901. 557p.

KENNEDY, Fiona; CLARKE, Sue; STOPA, Lusia, et al. Towards a cognitive model and measure of dissociation. J Behav Ther Exp Psychiatry. Mar 35(1), pp25-48. 2007. Disponível em: https://pubmed.ncbi.nlm.nih.gov/15157816/. Acesso em 03/08/2020. 
KORZEKWA, Marilyn; DELL, Paul; LINKS, Paul; THABANE, Lehana; FOUGERE, Philip. Dissociation in borderline personality disorder: A detailed look. Journal of Trauma \& Dissociation. 2009;10(3), pp346-367. Disponível em: https://pubmed.ncbi.nlm.nih.gov/19585341/. Acesso em 09/08/2020.

KRAUZE-UTZ, Annegret; FROST, Rachel; WINTER, Dorina \& ELZINGA, Bernet. Dissociation and alterations in brain function and structure: Implications for borderline personality disorder. Current Psychiatry Reports. 2017 Jan 19(1). Disponível em: https://pubmed.ncbi.nlm.nih.gov/28138924/. Acesso em 15/07/2020. (2017a)

KRAUZE-UTZ, Annegret; WINTER, Dorina; SCHRINER, Friederike; CHIU, Chiu-De; LIS, Stefanie; SPINHOVEN, Philip ... ELZINGA, Bernet. Reduced amygdala reactivity and impaired working memory during dissociation in borderline personality disorder. European Archives of Psychiatry and Clinical Neuroscience. 2018 Jun 268(4), pp401-415. Disponível em: https://pubmed.ncbi.nlm.nih.gov/28526931/. Acesso em: 15/07/2020. (2017b)

LINEHAN, Marsha. Treinamento de habilidades em DBT: Manual de terapia comportamental dialética para 0 terapeuta, $2^{\mathrm{a}}$ ed. Porto Alegre: Artmed, 2017. $528 \mathrm{p}$.

NEGRO JUNIOR, Paulo Jacomo; PALLADINO-NEGRO, Paula; LOUZÃ, Mario Rodrigues. Dissociação e transtornos dissociativos: modelos teóricos. Brazilian Journal of Psychiatry, Dez 1999 21(4), pp239-248. Disponível em: https://www.scielo.br/j/rbp/a/8rrPDMV98fQBGKPGpLJsq9k/abstract/?lang=pt. Acesso em 22/07/2020.

PEC, Ondrej; BOB, Petr; SIMEK, Jakub; RABOCH, Jiri. Dissociative states in borderline personality disorder and their relationships to psychotropic medication. Neuropsychiatric Disease and Treatment, Volume $14 . \quad$ pp32533257. 23 de novembro de 2018. Disponível em: https://www.ncbi.nlm.nih.gov/pmc/articles/PMC6260192/. Acesso em 04/09/2020. 
SAJADI, Seyede Fateme; ARSHADI, Nasrin; ZAGAR, Yadolla; MEHRABIZADE HONARMAN, Mahnaz; HAJJARI, Zahra. Borderline Personality Features in Students: the Predicting Role of Schema, Emotion Regulation, Dissociative Experience and Suicidal Ideation. International Journal of High Risk Behaviors and Addiction, 2015 Jun 30;4(2):e20021. Disponível em: https://pubmed.ncbi.nlm.nih.gov/26401490/. Acesso em 03/09/2020.

SCALABRINI, Andrea;

CAVICCHIOLI, Marco;

FOSSATI, Andrea; MAFFEI, Cesare. The Extent of Dissociation in Borderline Personality Disorder: A Meta-Analytic Review. Journal of Trauma \& Dissociation. Jul-Sep 2017;18(4) pp522-543. Disponível em: https://pubmed.ncbi.nlm.nih.gov/27681284/. Acesso em 13/06/2020.

SKRYPINSKA, Dagna; SZMIGIELSKA, Barbara. Dream-reality confusion in borderline personality disorder: a theoretical analysis. Front. Psychol. 2015; 6: 1393.15 de setembro de 2015.2 Disponível em: https://www.ncbi.nlm.nih.gov/pmc/articles/PMC4569816/ Acesso em 02/09/2020.

WAINER, Ricardo et al. Terapia cognitiva focada em esquemas: Integração em psicoterapia. Porto Alegre: Artmed, 2016. 240 p.

Enviado: Janeiro de 2021.

Aprovado: Junho de 2021. 